\title{
Design considerations of a lower limb exoskeleton system to assist walking and load-carrying of infantry soldiers
}

\author{
Seungnam $\mathrm{Yu}^{\mathrm{a}, *}$, Changsoo $\mathrm{Han}^{\mathrm{b}}$ and Ilje Cho ${ }^{\mathrm{a}}$ \\ ${ }^{a}$ Remote System Technology Development Section, Korea Atomic Energy Research Institute, Deajeon, South Korea \\ ${ }^{\mathrm{b}}$ Department of Robot Engineering, Hanyang University, Ansan, South Korea
}

\begin{abstract}
This paper describes the development of a wearable exoskeleton system for the lower extremities of infantry soldiers and proposes appropriate design criteria based on existing case studies. Because infantry soldiers carry a variety of equipment, the interference with existing equipment and additional burden of the exoskeleton support system should be minimized. Recent studies have shown that a user only needs to be supported in the gravitational direction when walking on flat terrain; however, active joints are necessary to support walking over rough and sloped terrain such as mountains. Thus, an underactuated exoskeleton system was considered: passive joints are applied to the hip and ankle joints, and active joints are applied to the knee joints to exploit the dynamic coupling effect of the link structure and muscular activation patterns when the user is going up and down stairs. A prototype of the exoskeleton system was developed and validated through a simple stair-climbing experiment.
\end{abstract}

Keywords: Exoskeleton, wearable robot, infantry soldier, underactuated system, weight suppot mechanism (WSM)

\section{Introduction}

According to records from the past 10 years, heavy personal belongings on soldiers have increased [1, 2]. This inevitably increases the possibility of musculoskeletal diseases; likewise, injuries to soldiers in circumstances other than battles, i.e., nonbattle injuries, have emerged as problems that critically affect the physical condition of soldiers [3-6]. The increase in these heavy personal belongings results from individual soldiers' requests to be sufficiently equipped to ensure personal safety in any circumstance as well as the increase in additional equipment used during field operations. For example, the maximum weight of personal belongings recommended for a U.S. infantry soldier is known to be approximately $23 \mathrm{kgf}$, whereas

*Corresponding author: Seungnam Yu, Remote System Technology Development Section, Korea Atomic Energy Research Institute, Deajeon, South Korea. E-mail: hymecer@gmail.com. the practical weight of personal belongings is known to reach no less than 45-60 kgf [1]. This tendency is true for other countries' infantry systems as well; thus, it is necessary to improve transportation equipment for heavy personal belongings, to minimize nonbattle injuries such as musculoskeletal diseases and to enhance the fighting power and mobility of infantry soldiers. In particular, in the field of national defense, many wearable lower extremity exoskeleton systems have been proposed, focusing on relieving the weight of the backpack, which is one of the major heavy personal belongings of individual wearers [7-11]; some of these systems emphasize their effectiveness by measuring the metabolic rate changes of wearers [12, 13]. However, as proposed in some studies, these systems tend to hinder the natural gaits of wearers owing to the excessive application of actuators or the kinematic problems of exoskeletons, and this tendency is a primary cause of the additional metabolic consumption of wearers [14]. Considering the nature of infantry 
duties in open fields, natural walking and running are essential elements; thus, the exoskeleton system these soldiers wear should be designed to avoid hindering their natural walking and to relieve the weight of heavy personal belongings. This study analyzes and proposes various design requirements through existing research and development cases for implementing the wearable exoskeleton system for infantry soldiers. Furthermore, a muscular power assistance system for offsetting muscular stress is proposed to attribute to the weight of personal belongings. This study focuses on the weight-supporting mechanism (WSM) for major heavy personal belongings such as backpacks while addressing a general wearable lower-extremity muscular power assistance system. The conclusion proposes requirements for improving personal portable equipment related to the exoskeleton system for infantry soldiers.

\section{Consideration of the motional characteristics of human bodies and proposal of the exoskeleton joint structure}

\subsection{Outline}

Because the infantry is a branch of the military service in which soldiers primarily conduct their duties in open fields, superior strength and exercise capacities are required. In particular, regarding exercise capacity, various motions such as walking and running erect, sitting on the knees, and crawling are required according to behavior types such as shooting, moving, and equipment handling. It is critical that the exoskeleton system not restrict these behaviors. Although the detailed structure will be discussed in Section 3, the exoskeleton system, when various motions are performed as shown in Fig. 1, should not hinder the motions of wearers. Consequently, structurally specific parts should not excessively protrude nor weigh heavily, and the system should be easily worn and removed.

\subsection{Two-jointed muscle}

Prior to determining the actuating structure of the exoskeleton system, the characteristics of the human body's muscles should be considered. Generally, flexors and extensors belonging to the same joint can also function as the flexors and extensors of the adjacent joint. These muscles are designated two-jointed muscles (TJMs) and are sometimes called biarticu- late muscles or biaxial muscles (Fig. 2). These TJMs, which are formed over two joints, are distributed over shoulders, elbows, hip joints, knee joints, and ankle joints and enable human bodies to generate various and complicated motions. Among these joints, this study concentrates on hip joints. Joints such as semitendinosus, semimembranosus, and biceps femoris (long head) are simultaneously involved in hip joint extension and knee flexion. In other words, the simple act of raising a straightened leg requires the interaction of various muscles between hip joints and knee joints. Thus, the simple application of actuators to hip joints/knee joints/ankle joints for the exoskeleton system, which is operated in complicated open fields, may possibly hinder the wearers' motions and movements.

In particular, active exoskeleton joints applying actuators require synchronizing signals from the wearers for actuation. However, in field operations, sensors receiving these synchronizing signals, irrespective of the human body's contact methods (i.e., electromyogram sensors or muscle stiffness sensors) or indirect/noncontact methods (i.e., force sensors, pressure sensors attachable to shoes, acceleration sensors for each link, encoder for each joint $[19,20])$, cannot infer all of the wearers' intentions for complicated motions. In particular, in the case of electromyogram sensors, most studies have examined using electric signals individually extracted from each muscle; as previously stated, because TJMs play an important role in the maneuvers performed by an infantry soldier, if electromyogram signals are used, signals from various muscles should be comprehensively analyzed for practical use.

\subsection{Joint composition of the lower extremity exoskeleton system}

Of the various lower extremity exoskeleton systems introduced in recent years, the BLEEX system of U.C. Berkeley, HULC of Lockheed Martin, and EXO series of Sarcos are widely known military exoskeleton systems for infantry soldiers [7-10]. Further studies have been continuously conducted on these systems, and the overall direction of improvement can be defined as the simplification of the exoskeleton and actuator system. In other words, the current independent source technology lacks technical maturity for applying an exoskeleton system using a number of high mobility actuators for individual soldiers; in particular, it is difficult to persuade soldiers who wear various personal 

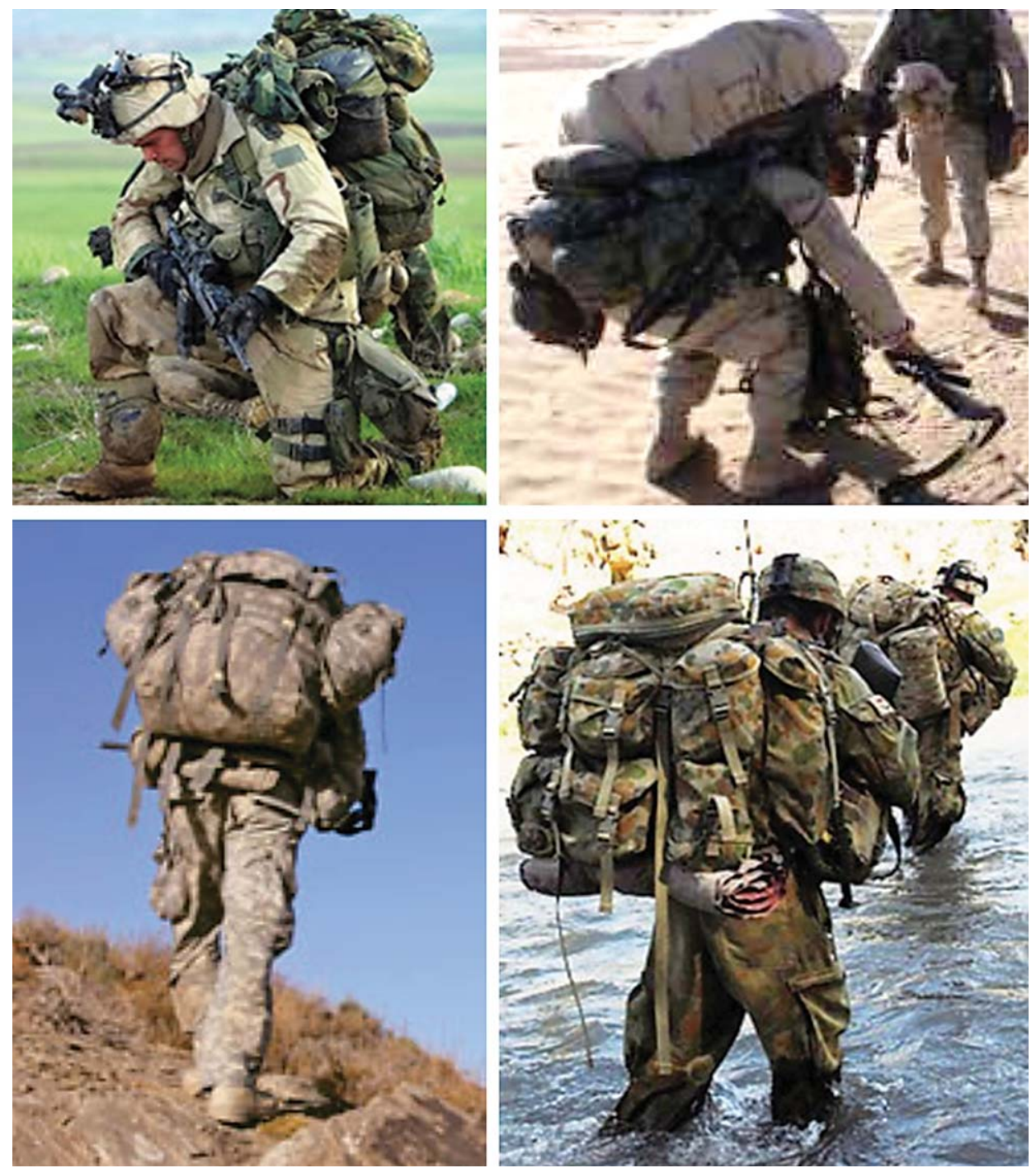

Fig. 1. Field operations of infantrymen in carrying loads [15-18].

portable equipment to wear an additional heavy exoskeleton system, even when considering advantages such as supporting the weight of heavy personal belongings. Considering these overall limitations, the actuating method for the exoskeleton system used in open fields is proposed as follows.

- As exoskeleton joints corresponding to joints directly related to TJMs (hip joints and ankle joints), passive, or quasi-passive joints can be applied; for the application of active joints, the actuating mechanism of actuators needs to have compliance or backdrivability.
- The overall exoskeleton system should distribute the weight of the backpack to body parts below the waist and simultaneously support the weight to minimize the weight burden on the wearer. At this time, the mechanism for weight support should simultaneously function as passive hip joints.

Based on these considerations, this study proposes an underactuated system as an exoskeleton system for infantry soldiers, implementing passive joints for hip joints to enable walking and the WSM for backpacks, active joints using an electric motor for knee joints, and passive joints using an elastic body for ankle joints. For 

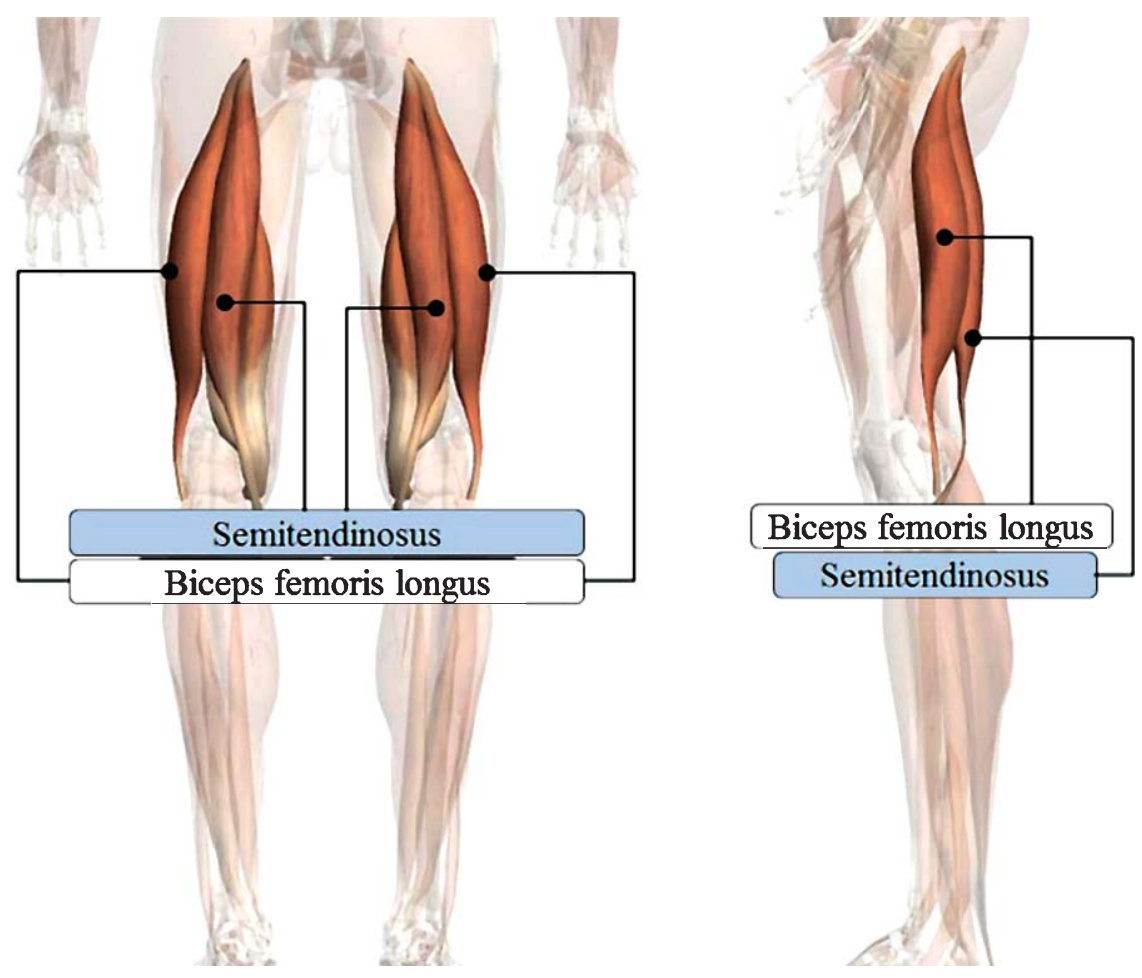

Fig. 2. Two-jointed muscle for hip joint.

knee joints, rotating and linear actuators were variously applied based on existing studies, and cases in which rotating active joints were used (i.e., previous studies) are given as references [21]. Sensing of wearers' motion intentions for actuating active joints will not be discussed in this study. However, since it is difficult to measure bio-signals using an electromyogram sensor because infantry soldiers operate in open fields, physical sensors such as pressure, and acceleration sensors are considered to be appropriate.

\subsection{Intersegmental dynamics}

Infantry soldiers frequently traverse slopes such as mountains to conduct operations. The passive joint exoskeleton system [22] for walking on flat land and supporting the weight of backpacks, which was proposed in a recent similar study, cannot assist muscular power when moving on slopes; this is a result of focusing on lightening and supporting the weight of the system. In this respect, systems such as the underactuated system proposed in this study can be used on sloped terrains by supporting the wearers' muscular power and the weight of backpacks and ensuring a moderate wearing sensation. In terms of system efficiency, the placement of the optimized actuators is particularly important, which implies that, as previously stated, the placement of actuators on the exoskeleton (passive hip joints, active knee joints, and passive ankle joints) needs to be considered in terms of muscular activation characteristics and dynamics. According to existing studies on the body's muscular functions related to walking, the motion of lifting heels when overcoming an obstacle or moving up stairs consists of bending hip joints, knee joints, and ankle joints. At this time, the energy transferred to each joint is appropriately adjusted according to the heights of obstacles or stairs. The motions of bending hip and ankle joints are similar to passive motions, which are caused more by the active force of these joints than by the motion of rotating knee joints. This segmentation of joint moment can be analyzed as a dynamical optimization method based on the interaction between segments of the human nervous system to minimize energy consumption related to motions such as overcoming obstacles or moving up stairs [23]. This is also verified by the dynamical coupling effect in the underactuated systems [24, 25]. In other words, it can be 
inferred that a certain level of muscular power assistance with respect to hip joints can be achieved only through the actuating power of the knee joints. In this respect, as proposed in existing studies, experimental results indicate that the overall muscular power assistance for major activated muscles of wearers' lower extremities with respect to the motion of going up stairs (Figs. 11 and 12) can be achieved only by actuating the exoskeleton knee joints [21].

\section{Kinematic structure of an exoskeleton system for infantry soldiers}

\subsection{Leg structure}

In this section, the structure related to the link shape of the lower extremity exoskeleton system is considered. If $L_{\text {Thigh }}$ is defined as the length of the thigh and $L_{\text {Shin }}$ as the length of the shin of the exoskeleton, the leg length of the exoskeleton is advantageous in the following proportions.

$$
\frac{L_{\text {Thigh }}}{L_{\text {Shin }}}<1
$$

This is because the exoskeleton creates swings in the wearer's lower extremity on which the exoskeleton is attached; at this time, the overall trajectory of these swings depends on the trajectory of the wearer's feet. In other words, when regarding the feet of the exoskeleton as the end-effector of the manipulator, the thigh and shin parts correspond to the 2-link manipulator; the swing of lower extremities during the swing phase of general walking cycles shows the process from the initial swing section to the landing point, similar to the motion of drawing a circular arc with a full-stretched arm. At this time, in terms of manipulability, the structure in Equation (1) can gather higher speed with the same energy, and in terms of wearers, more natural walking can be implemented with less force. This is also advantageous for the military purpose in terms of the efficiency of walking and stride control while connected to the exoskeleton [26]. Consequently, the B-type structure is more advantageous than the A-type structure, as shown in existing study cases in Fig. 3.

\subsection{Mechanism of ankle joints}

The mechanism of ankle joints of the exoskeleton, in general cases, is an important linking point between the wearer and the exoskeleton; additionally, it comprehensively supports heavy personal belongings and the weight of the exoskeleton itself. In previous studies [21], by using the method mentioned in Section 2, passive ankle joints, which are highly elastic and composed of hyper carbon that can withstand the weight of the entire upper part, were applied. This enables wearers to perform an auxiliary function for natural forward walking by emitting energy, which is stored by the elastic deformation of the walking mechanism in the process of heel contact after the swing section, during the stance and toe-off sections. In recent studies, a separate actuator has not been used for ankle joints; in some systems, the stiffness of ankle joints can be adjusted. It is known that ankle joints can adjust elasticity, and in particular, in the case of the underactuated system, they can also perform tuning functions such as adjusting the motion area of other adjacent passive joints [22]. Consequently, the ankle joints of the exoskeleton can exhibit sufficient performance with the following functions: 1) the linking point to wearers; 2) the transfer of the weight of the upper exoskeleton and the external heavy personal belongings to the ground; 3) the provision of a stable wearing sensation by appropriately limiting the turning force or converting energy; and 4) the support of momentum.

\subsection{WSM for backpacks}

\subsubsection{Outline}

Backpacks have the heaviest weight among the personal portable equipment of general soldiers. The lower extremity exoskeleton also has the primary purpose of supporting the weight of backpacks. In the case of this WSM, the weight support by applying the active joints for hip joints can be considered; however, as previously stated, we propose a method to apply a WSM that can simultaneously perform the functions of supplying passive joints for the hip joints of the exoskeleton and of supporting the weight of backpacks. Various WSMs for backpacks have been proposed since the early $2000 \mathrm{~s}$ for national defense applications. Recently, as introduced in the previous section, WSMs with a planar linkages have been proposed [21, 22]. However, even these systems show differences in function according to whether the active adjustment of stiffness is possible; in particular, the system proposed by Hollander actively adjusts the stiffness by using control signal patterns, which are calculated in response to the weight of backpacks and walking 


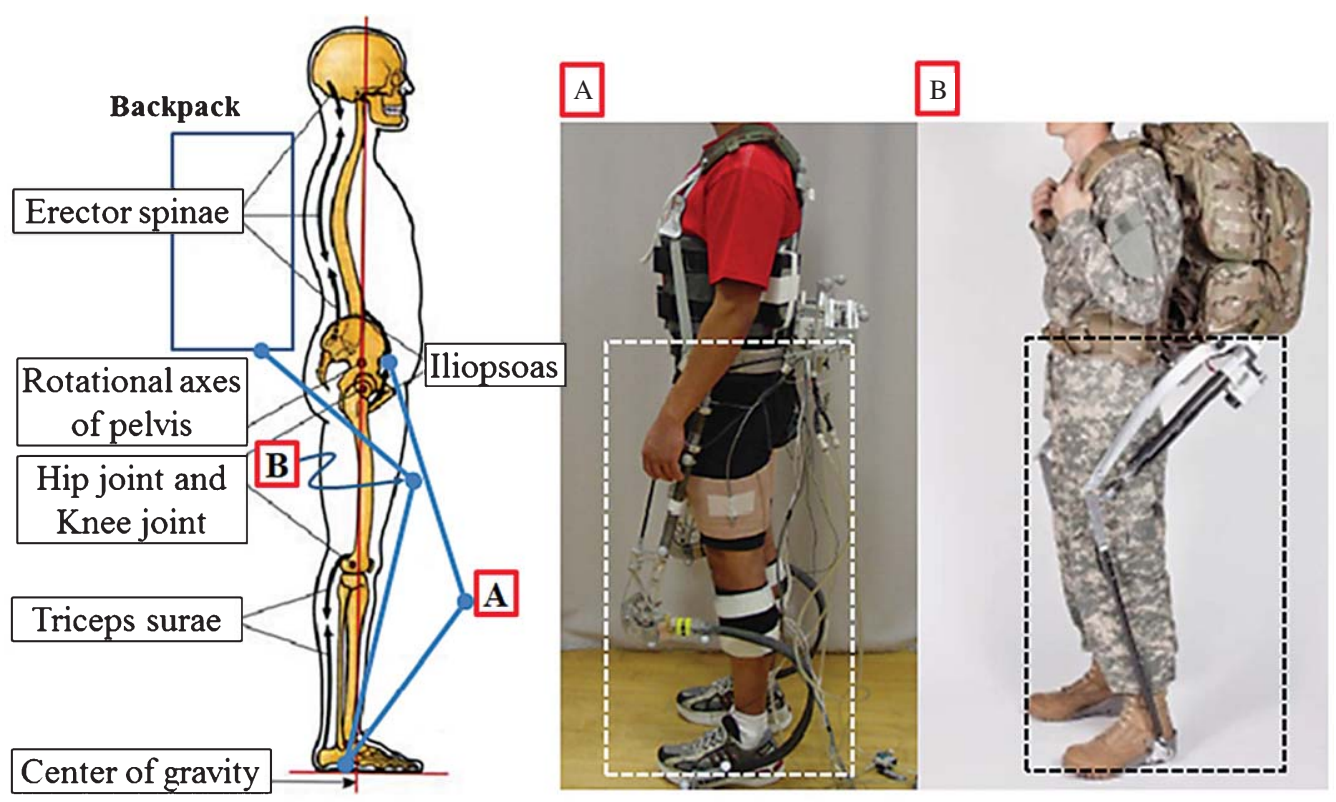

Fig. 3. Comparison of linkage structures of exoskeleton systems [22, 27].

cycles of the wearers [22], and a separate external electrical power is installed for this purpose. In addition, the system proposed by Yu was designed to manually adjust the stiffness of springs according to the weight of wearers' heavy personal belongings and their individual preferences for motion range while walking; thus, a separate external electrical power is not required (Of course, this kind of system used an external power to operate the actuator of an active joint.). In particular, in the latter case, the stiffness was selected and applied by the constant force mechanism (WSM) analytical method based on springs; by using this method, the mechanism itself was maintained in a constantly deformed posture in response to the external weight (the weight of backpacks) [21] (Fig. 4).

Generally, the frequency of jogging is approximately $3 \mathrm{~Hz}$ per s [28]. If this applies to soldiers in full gear, backpacks vibrate approximately 3 times per second while running. On the other hand, the vertical ground reaction force is known to be approximately 2.5 times greater than their weight; if this applies to backpack wearers, the impulsive-weight approximately 2.5 times greater than the weight of the backpack is loaded on their shoulders when they land on the ground while walking [28]. An existing analytic study of carrying poles indicated that a considerable amount of the weight loaded on the shoulders can be reduced by comparing the vibration frequency of heavy personal belongings, which are supported by the shoulders, with the human body's vertical frequency while walking [28]. This can be applied to the wearable lower extremity exoskeleton system based on the WSM by adjusting the stiffness of this system and, therefore, the vibration frequency of backpacks. Of course, when adjusting the stiffness using this system, it should be tuned in response to each case by using various wearing tests of the vibrations of backpacks while wearers are walking and running.

\subsubsection{Selection of the spring specifications of the WSM}

To implement the WSM proposed in this study, a widely known spring design method is used [29]. When springs are deformed, shear force, $F$, and torsional moment, $T$, are produced in the cross section, as shown in Fig. 5. At this time, the maximum stress loaded on the wire represents the overlapped value of shear stress by direct shear stress and torsional moment. If the average diameter of springs and the diameter of wire composing the springs are defined as $D$ and $d$, respectively, this can be expressed by the strain energy analytical method as follows: 


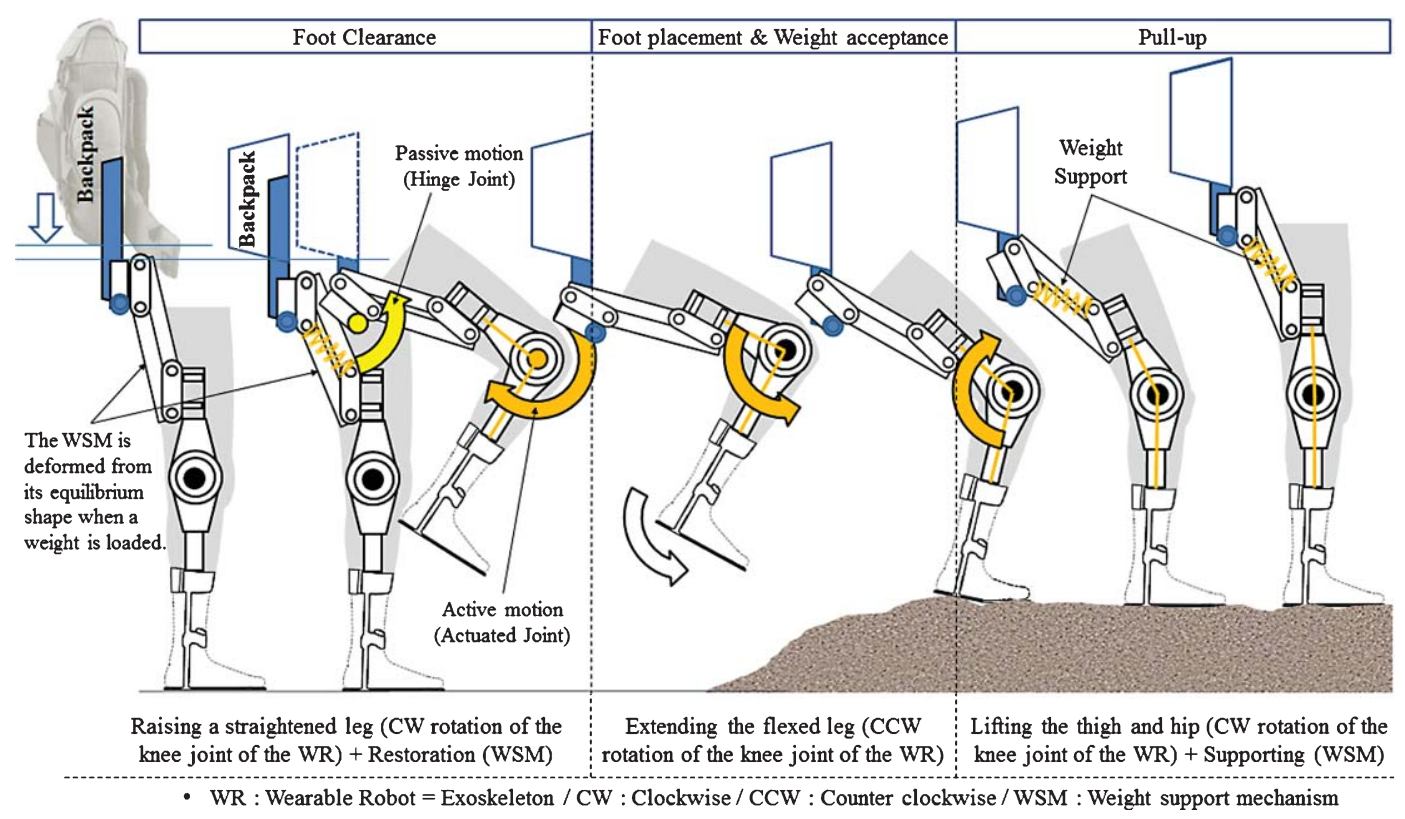

Fig. 4. Motion strategy of the designed exoskeleton system for walking and load-carrying in the field condition.
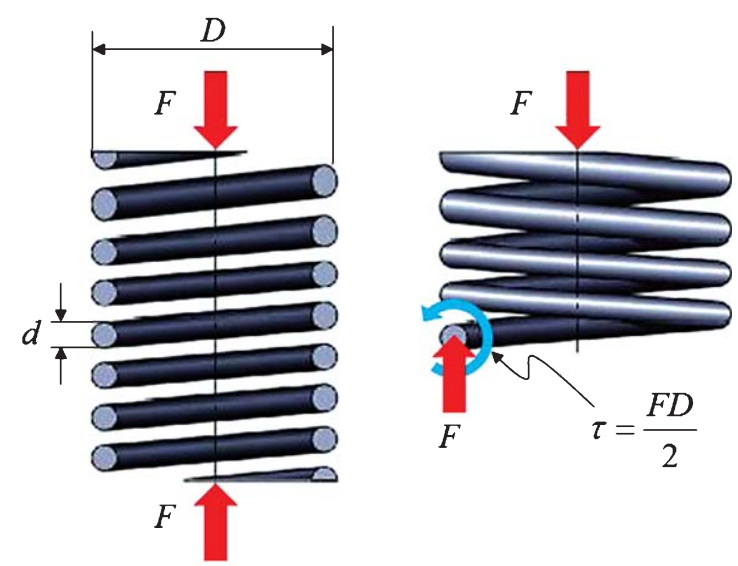

Fig. 5. Spring design parameters.

$$
E_{S}=\frac{F^{2} l}{2 A G}+\frac{T^{2} l}{2 G J}
$$

Here, $A$ is the cross-sectional area and $G$ is the shear modulus.

Here, if the variables in Equation (2) are defined as in Equation (3), we can obtain Equation (4).

$$
T=\frac{F D}{2}, l=\pi D N_{a}, J=\frac{\pi d^{4}}{32}, A=\frac{\pi d^{2}}{4}
$$

$$
E_{S}=\frac{2 F^{2} D N_{a}}{d^{2} G}+\frac{4 F^{2} D^{3} N_{a}}{d^{4} G}
$$

$N_{a}$ in Equations (3) and (4) represents the number of spring coils that are practically deformed in the springs and $l$ is the length of the spring wire. If the total deformation of springs is defined as $r$, the following equation can be derived:

$$
r=\frac{\delta E_{S}}{\delta F}=\frac{4 F D N_{a}}{d^{2} G}+\frac{8 F D^{3} N_{a}}{d^{4} G}
$$

If we assume that the second term is greater than the first term, Equation (5) can be simplified as follows:

$$
\begin{gathered}
r \cong \frac{8 F D^{3} N_{a}}{d^{4} G}=\frac{F}{k_{s}} \\
k_{s}=\frac{d^{4} G}{8 D^{3} N_{a}}
\end{gathered}
$$

As shown in the equations above, the stiffness of springs $k_{s}$ is related to the number of spring coils as well as the external structure, such as the spring size. This finding has been used for various methods of exoskeleton and weight-supporting systems. In other words, it is known that the stiffness of springs can be modified by adjusting the number of spring coils, or by changing the shape of the springs $[30,31]$. 


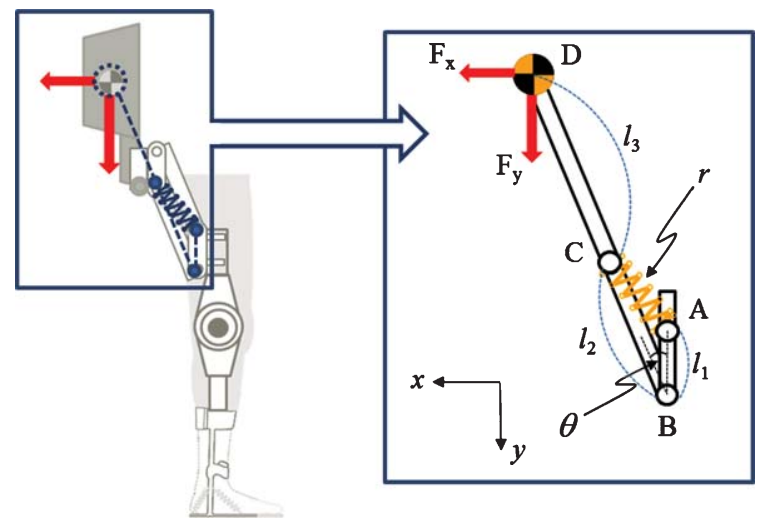

Fig. 6. Kinematic structure of the WSM.

In the remaining sections of this chapter, mathematical approach will be presented for the design of the weight support mechanism for the proposed exoskeleton system.

Figure 6 shows the mechanical assumption and modeling. The gravitational force induced by the mass of the backpack is applied at $D(x, y)$, and the base frame is located at point $\mathrm{B}$. The position of point $D$ is expressed as follows:

$$
\begin{gathered}
x=\left(l_{2}+l_{3}\right) \sin \theta \\
y=l_{1}-\left(l_{2}+l_{3}\right) \cos \theta
\end{gathered}
$$

Points $\mathrm{A}$ and $\mathrm{C}$ are connected by a linear spring; its length is expressed by

$$
r^{2}=l_{1}^{2}+l_{2}^{2}-2 l_{1} l_{2} \cos \theta
$$

The performance at point $\mathrm{D}$ can be expressed by using $r$ for each axis. Differentiating Equation (10) with time produces

$$
r \dot{r}=l_{1} l_{2} \sin \theta \dot{\theta}
$$

From Equations (8) and (9),

$$
\begin{gathered}
\dot{\theta}=\frac{\dot{x}}{\left(l_{2}+l_{3}\right) \cos \theta} \\
\sin \theta=\left[\frac{\left(2 l_{1} l_{2}\right)^{2}-\left(l_{1}^{2}+l_{2}^{2}-r^{2}\right)^{2}}{4 l_{1}^{2} l_{2}^{2}}\right]^{\frac{1}{2}}
\end{gathered}
$$

After Equations (12) and (13) are substituted into Equation (11), the time-dependent displacement relation between point $\mathrm{D}$ and spring length $l_{s}$ for the $x$ axis is

$$
\begin{aligned}
\dot{x}= & {\left[\frac{\left(l_{2}+l_{3}\right)\left(l_{1}^{2}+l_{2}^{2}-r^{2}\right)}{2 l_{1}^{2} l_{2}^{2}}\right.} \\
& \left.\left\{\frac{4 l_{1}^{2} l_{2}^{2}-\left(l_{1}^{2}+l_{2}^{2}-r^{2}\right)^{2}}{4 l_{1}^{2} l_{2}^{2}}\right\}^{-\frac{1}{2}} r\right] \dot{r}
\end{aligned}
$$

Briefly,

$$
\begin{gathered}
\dot{x}=g_{r}^{x} \dot{r} \\
\because g_{r}^{x}=\frac{\left(l_{2}+l_{3}\right)\left(l_{1}^{2}+l_{2}^{2}-r^{2}\right)}{2 l_{1}^{2} l_{2}^{2}} \\
\left\{\frac{4 l_{1}^{2} l_{2}^{2}-\left(l_{1}^{2}+l_{2}^{2}-r^{2}\right)^{2}}{4 l_{1}^{2} l_{2}^{2}}\right\}^{-\frac{1}{2}} r
\end{gathered}
$$

Likewise for the $y$-axis,

$$
\begin{gathered}
\dot{y}=\left[\frac{\left(l_{2}+l_{3}\right)}{l_{1} l_{2}} r\right] \dot{r} \\
\dot{y}=g_{r}^{y} \dot{r} \\
\because g_{r}^{y}=\frac{\left(l_{2}+l_{3}\right)}{l_{1} l_{2}} r
\end{gathered}
$$

These equations can be used to derive the effective stiffness. At point D, the effective force $F_{\text {eff }}$ is expressed as follows based on the virtual work theory.

$$
F_{e f f}=F_{s}+g_{r}^{x} F_{x}+g_{r}^{y} F_{y}
$$

Here, the effective stiffness of the applied spring is defined as follows:

$$
\begin{aligned}
k_{s}= & -\frac{\partial F_{e f f}}{\partial r}=-\frac{\partial F_{s}}{\partial r}-g_{r}^{x} \frac{\partial F_{x}}{\partial r}-g_{r}^{y} \frac{\partial F_{y}}{\partial r} \\
& -h_{r}^{x} F_{x}-h_{r}^{y} F_{y}\left(\because h_{r}^{x}=\frac{g_{r}^{x}}{\partial r}, \quad h_{r}^{y}=\frac{g_{r}^{y}}{\partial r}\right)
\end{aligned}
$$

For simplification, we assume that the external force exerted on the spring mechanism is from the loaded weight on the backpack and that there is no ducking motion while the wearer operates this system. Of course, ducking motion can be performed in the field operation, and this system still support the gravitational force of the mass of the backpack decomposed along the axis parallel to the wearer's back. However, another force of the mass decomposed along the axis vertical to the wearer's back have to be borne by wearer's own 
muscle force. It would be presented in the next chapter. For the standing posture, the exerted forces on each axis are as follows:

$$
\begin{gathered}
F_{x}=0 \\
F_{y}=\text { constant }
\end{gathered}
$$

If the initial length of spring is considered as $r_{0}$, the elastic force is

$$
F_{s}=-k_{s}\left(r-r_{0}\right)
$$

The effective force of Equation (20) is expressed in Equation (25) using Equations (19, 20, 22 and 24).

$$
F_{e f f}=-k_{s}\left(r-r_{0}\right)+\frac{\left(l_{2}+l_{3}\right)}{l_{1} l_{2}} r F_{y}
$$

To ensure stable walking while the system is loaded with the allowable weight, the additional condition of $F_{\text {eff }}=0$ should be added.

$$
k_{s} r_{0}=\left(k_{s}-\frac{\left(l_{2}+l_{3}\right)}{l_{1} l_{2}} F_{y}\right) r
$$

Therefore, the equilibrium position of the spring mechanism while loaded with weight is calculated as follows:

$$
r=\frac{k_{s} r_{0}}{k_{s}-\frac{\left(l_{2}+l_{3}\right)}{l_{1} l_{2}} F_{y}}\left(k_{s} \neq \frac{\left(l_{2}+l_{3}\right)}{l_{1} l_{2}} F_{y}\right)
$$

Selecting the stiffness of the designed spring mechanism involves a tradeoff between the stability of loaded weight and comfort of the wearer's leg swing. Adequate stiffness of the designed system is confirmed by using Equation (27) to maximize the allowable weight. In practice, a wearer can modify the stiffness by adjusting the spring mechanism while wearing the system. Finally, substituting Equation (7) into Equation (27) gives the following relationship between the spring parameter of the WSM and the external load of the exoskeleton:

$$
\left(\frac{d^{4} G}{8 D^{3} N_{a}}\right)\left(\frac{l_{1} l_{2}}{l_{2}+l_{3}}\right)\left(1-\frac{r_{0}}{r}\right)=F_{y}
$$

\subsubsection{Weight balance problem while carrying the backpack}

In the case of passive exoskeleton joints of hip joints, the moment is produced by the eccentricity with respect to the center of the human body when supporting heavy personal belongings such as backpacks; this phenomenon is often thought to adversely affect wearers' balance while standing erect and walking. However, the following simple calculations and actual experiments verify that this phenomenon is unlikely to happen. The angular acceleration of the heavy personal belonging of mass $M$ in Fig. 7 can be expressed as shown in Equation (29).

$$
\ddot{\theta}=-\frac{g}{L} \sin \theta
$$

Here, $g$ is the acceleration of gravity, $L$ is the distance from the center of gravity of the backpack to the center of a passive joint of the hip joint, and $\theta$ is the rotation angle of $\overline{B D}$ with respect to $\overline{A B}$.

The force and acceleration of mass $M$ is expressed by the analytical method in inverted pendulum model as follows:

$$
\begin{gathered}
M \ddot{x}=F_{x} \\
M \ddot{y}=F_{y}-M g \\
\ddot{x}=-L\left(\ddot{\theta} \cos \theta+\dot{\theta}^{2} \sin \theta\right) \\
\ddot{y}=L\left(\ddot{\theta} \sin \theta-\dot{\theta}^{2} \cos \theta\right)
\end{gathered}
$$

where $\dot{\theta}$ is the angular speed of mass $M$ with respect to passive joint $\mathrm{B}$ of the hip joint in Fig. 7. By substituting Equation (29) into Equations (32) and (33) and multiplying each equation by the mass $M$ of the backpack, the magnitude of the acting force by the backpack can be found. Finally, the component forces exerted on the point $\mathrm{B}$ of the backpack in the horizontal

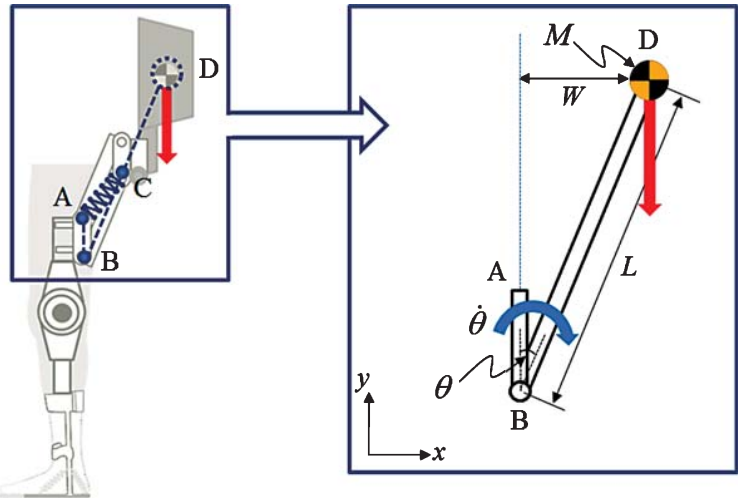

Fig. 7. Parameters of the WSM for the consideration of the weight balance problem. 
and gravitational directions can be derived respectively as follows:

$$
\begin{aligned}
-M \ddot{\theta} L \cos \theta-M \dot{\theta}^{2} L \sin \theta & =F_{x} \\
M \ddot{\theta} L \sin \theta-M \dot{\theta}^{2} L \cos \theta+M g & =F_{y}
\end{aligned}
$$

These equations are expressed in Equations (34) and (35) are simplified as follows:

$$
\begin{aligned}
& M\left(g \cos \theta-L \dot{\theta}^{2}\right) \sin \theta=F_{x} \\
& M\left(g \cos \theta-L \dot{\theta}^{2}\right) \cos \theta=F_{y}
\end{aligned}
$$

If $M=40 \mathrm{~kg}, L=0.4 \mathrm{~m}$ and $W=0.2 \mathrm{~m}$ are substituted in the above equations, as the $W$ (or $\theta$ ) value decreases, force $F_{x}$ in the direction in which the backpack moves away from the wearer's back drops sharply, but on the other hand force $F_{y}$ in the direction of the acceleration of gravity increases steadily. Here, because $F_{y}$ is supported by the WSM of the hip joint, as the backpack adheres more closely to the wearer's back when its shoulder strap is tightened, $F_{y}$, which is produced by the weight of the backpack, is minimized; this means that the wearer can maintain the balance of the backpack with less force. In this respect, the distribution of weight inside the backpack, carrying heavy personal belongings and connected to the exoskeleton, is important $[32,33]$.

\section{Actuation strategy and selection of the actuator specifications for knee joints of the exoskeleton system}

To apply actuators to the knee joints of the exoskeleton system, it is necessary to determine the appropriate actuator specifications by comprehensively inferring and examining the circumstances faced by wearers. This is because the increase in required torque is followed by an increase in the weight of the actuators; consequently, the increased weight of actuators acts as an additional weight on the soldiers. In existing studies related to the selection of actuator capacity of the exoskeleton system, there are some cases in which the actuator torque capacity of the knee joints of the exoskeleton system was calculated while moving up stairs [34]. This study, in this respect, is intended to calculate the required torque of the human body's knee joints while moving up and down stairs in terms of vital dynamics, and to propose a method for transferring power to the exoskeleton system by using the selected actuator based on the calculation.

Existing studies indicate that the dorsi/planar flexion of ankles does not absorb a large amount of energy in the initial stepping motion section while moving up and down stairs and requires a large amount of force in the latter support section [35]. Consequently, the average dorsi/planar flexion power of ankles increases slightly while going up and down stairs than while walking on flat land. However, in the case of knee joints, the widest difference in required force from that while walking on flat land occurs while moving up and down stairs; at this time, the instantaneous maximum force capacity is known to be approximately $200 \mathrm{~W}$. When compared to $30 \mathrm{~W}$ when walking on flat land, this is a wide variation. The required torque in the swing section is similar to that while walking on flat land. The required force of tibiofemoral joints can be estimated by analyzing photographs and films using a stroboscope for measuring the number of rotations, and typically by analyzing [36] the swing speed data of lower legs based on dynamic activities such as ball kicking. According to related studies, the maximum acceleration at the instant of kicking a ball is $453 \mathrm{rad} / \mathrm{s}^{2}$, the mass moment of inertia of lower legs can be determined to be 0.35 $N m s^{2}$ from separate data on the human body [37], and consequently, the torque of tibiofemoral joints can be calculated as follows:

$$
0.35 \mathrm{Nms}^{2} \times 453 \mathrm{rad} / \mathrm{s}^{2} \cong 158.5 \mathrm{Nm}
$$

In addition to the torque, calculated by assuming that the distance from the patellar tendon to an arbitrary central point with respect to the tibiofemoral joint is $0.05 \mathrm{~m}$, the muscular force of the knee joint, $f_{\text {knee }}$, which acts through the patellar tendon, can be calculated as follows (Fig. 8):

$$
\begin{aligned}
& 158.5 \mathrm{Nm}=f_{\text {knee }} \times 0.05 \mathrm{~m} \\
& \therefore f_{k m}=3170 \mathrm{~N}
\end{aligned}
$$

The above value is regarded as the force for implementing the maximum rotational speed of the knee joint in the swing section. Thus, the required torque of the knee joint of the exoskeleton for supporting the muscular power of legs while climbing stairs is the value obtained by subtracting the torque reaction force in the gravitational direction, which acts on the upper legs by the ground reaction force while stepping on the ground, from the torque by the patellar tendon. 


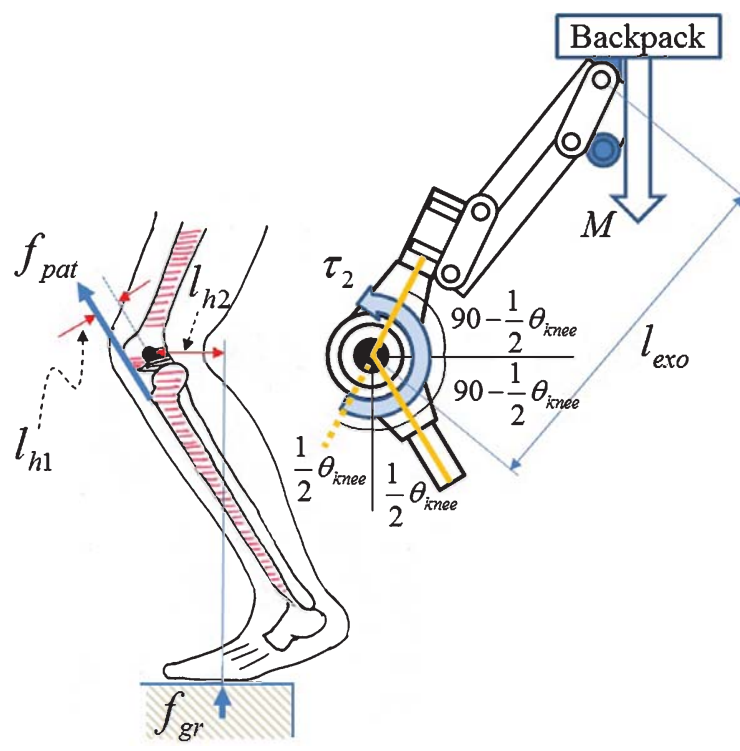

Fig. 8. Calculation of muscle force and external load for actuator selection.

We assumed maximum assistive force for the ground reaction force on each foot while climbing up the stairs as $750 \mathrm{~N}$ based on the considered wearer's own body weight. The moment of knee force is assumed that it is always larger than the one of the ground reaction force regardless of the wearer's weight and the phase of the walking cycle because there is a trade-off between the ground reaction force and its moment arm while stepping up motion. Therefore, the moment of the ground reaction force is considered to maintain a smaller value than the moment of the knee joint for the derivation of an effective required torque $\tau_{1}$ for knee support.

$$
\begin{aligned}
\tau_{1}= & f_{\text {pat }} \times l_{h 1}-f_{g r} \times l_{h 2} \\
& =3170 \mathrm{~N} \times 0.05 \mathrm{~m}-750 \mathrm{~N} \times 0.15 \mathrm{~m} \\
& \cong 50 \mathrm{Nm}
\end{aligned}
$$

Considering the previously mentioned studies and other figures related to the instantaneous torque of the human body's joints, the torque range can be determined in the range of 50-113 Nm. Here, additional torque is required for the sheer weight of the exoskeleton and the backpack. For a total weight (backpack, gears and one thigh segment of the exoskeleton system), $M$, of $65 \mathrm{~kg} f$ and a knee joint rotation angle, $\theta_{\text {knee }}$, of $40^{\circ}$, based on the parameters of Fig. 8 and estimated posture in phase transition from the 'weight

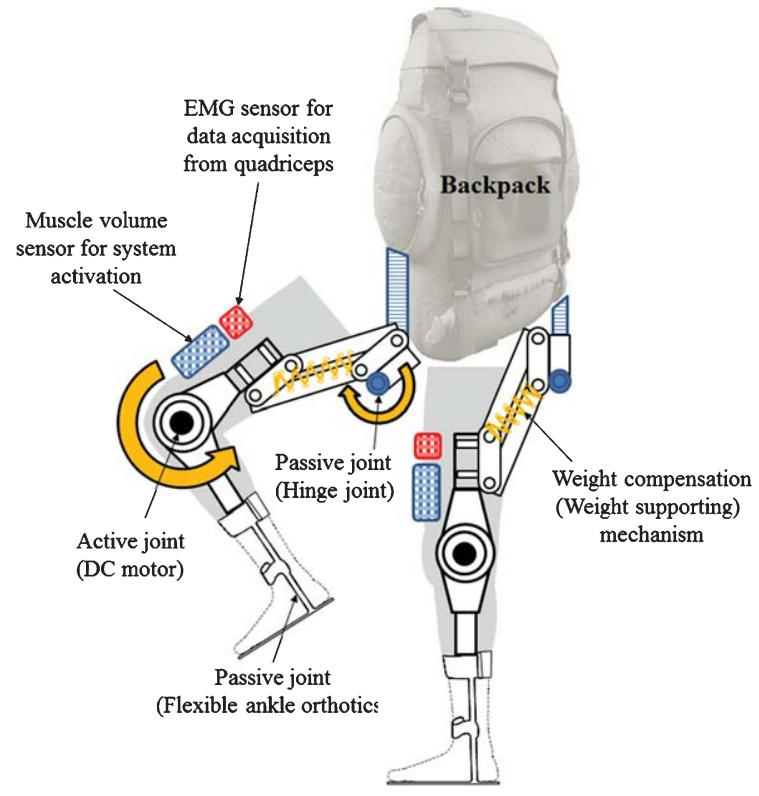

Fig. 9. Experimental setup of the proposed exoskeleton system.

acceptance' to the 'pull-up', illustrated in Fig. 12. The required torque of the exoskeleton knee joint, $\tau_{2}$, can be calculated as follows:

$$
\begin{aligned}
\tau_{2}= & M g l_{\text {exo }} \sin \theta_{\text {knee }} \\
& =650 \mathrm{~N} \times 0.5 \mathrm{~m} \times \cos \left(70^{\circ}\right) \\
& \cong 110 \mathrm{Nm}
\end{aligned}
$$

Hence, the final torque value of the exoskeleton, by combining Equations (40) and (41), can be calculated as the required value in the range of approximately $160 \mathrm{Nm}$. By combining the required speed value while moving up and down stairs (angular velocity of the knee joint), the actuator capacity (unit: $W$ ) to be applied to the knee joint of the exoskeleton can be determined.

\section{Manufacture of prototypes and experimental verification}

The exoskeleton system applying the design elements discussed in the previous section is implemented, as shown in Fig. 11; by using it, the validity of the proposed design elements is verified. The structure of the exoskeleton system proposed in this study and the result of the motion experiment of the muscle 

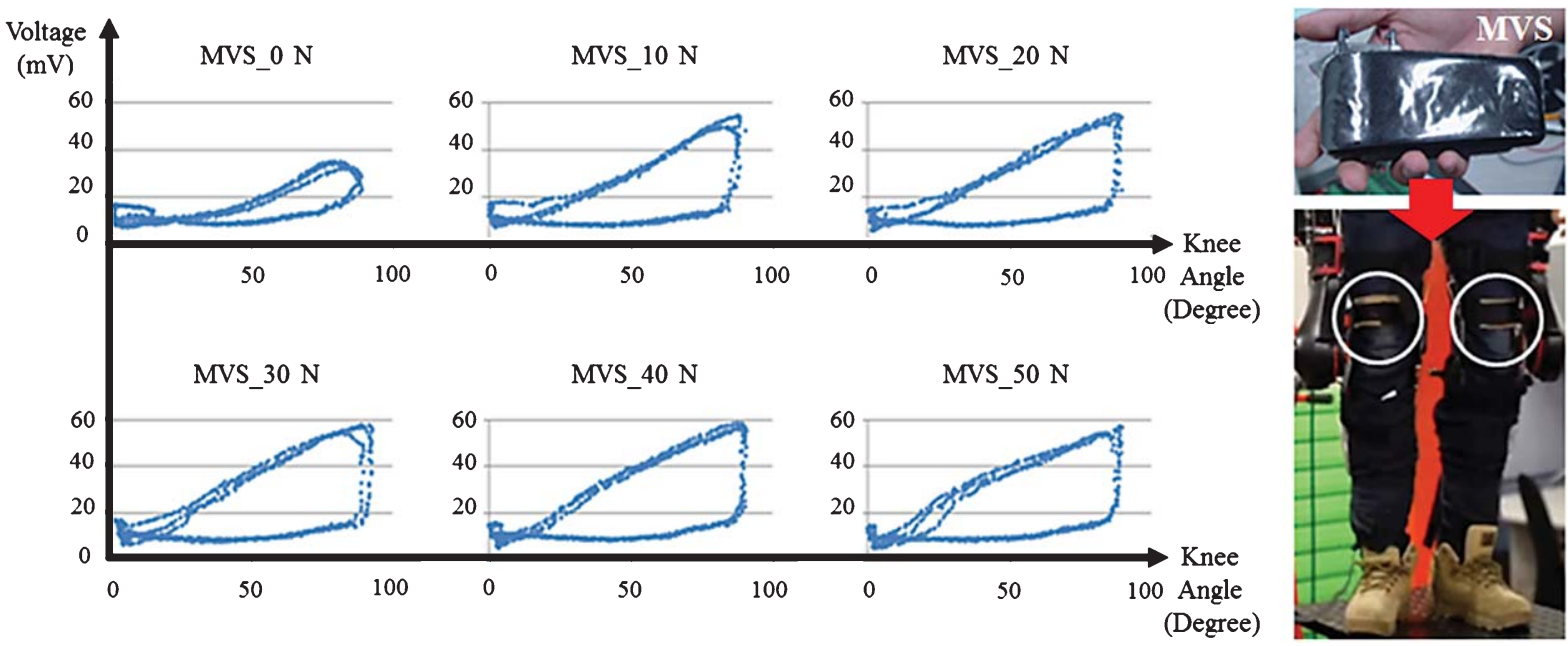

Fig. 10. Result of the performance test of the muscle volume sensor (MVS) applied to the exoskeleton system.

volume sensor (MVS) applied to the system are shown in Figs. 9 and 10, respectively. The MVS was designed to actuate the system through the wearer's intention of motion; detailed structure and motion principles of the MVS were omitted because they digress from the subject of this study. For further details, refer to the previous research of Seo [38]. The signal patterns of the MVS when performing an isotonic motion were determined by connecting a KINCOM ${ }^{\circledR}$, which measures the muscular power of each joint of the human body; the experimental subjects wearing the MVS to load the artificial external weight on their lower extremities are shown in Fig. 10. As shown in the figure, standardized signal patterns of a certain level were determined with respect to various types of external weight; these results are applicable to the exoskeleton system in this study, which addresses various types of heavy personal belongings. Scenes of performing the stair walking experiment, with the backpack of $40 \mathrm{kgf}$ installed in the system proposed in Fig. 9, are shown in Fig. 11. The measured muscular activities of the wearer before and after wearing the exoskeleton in respective walking sections, measured by using an electromyography (EMG) sensor attached in the vicinity of the quadriceps of the wearer's thigh, are compared in Fig. 12.

When wearing the exoskeleton, certain activated muscles can be identified in the swing phase section of $2-3 \mathrm{~s}$; this may have been affected by the fact that the thigh of the exoskeleton is lifted to some degree by the wearer's own muscular power because the hip joint of the exoskeleton is a hinge joint, which has no separate power source. Of course, the state of tension of muscles occurring during the wearer's learning process at the initial stage of walking after wearing the exoskeleton also had some effect: the muscular activity significantly dropped during the section of 5-6s, in which the same motions are performed with respect to the same swing motion performed during the section of 2-3 s. Consequently, as the wearer passed 1 to 2 cycles of walking and adapted himself to the system, which provided a certain level of muscular power assistance for the hip joint only by actuating the motor of the knee joint through the dynamic coupling effect, which was mentioned in Subsection 2.4, could simultaneously drop the overall muscular activity peak of the quadriceps to less than half the original level. In other words, it is thought that the lower extremity exoskeleton structure in this study, which combines the WSM installed in the hip joint, the motor-actuated joint of the knee joint, and the elastic body of the ankle joint, has sufficient validity.

Muscle activation for the subjects using the developed exoskeleton was fully measured and analyzed in the previous report already. The report included full images of one-cycle climbing with the exoskeleton, RMS data of measured EMG signals, \%MVIC of subjects' muscles with/without the exoskeleton [21]. Thus, this paper additionally emphasized the result of intersegmental, and user adaptation transition effects of the proposed exoskeleton while performing the weight supporting in Fig. 12. 

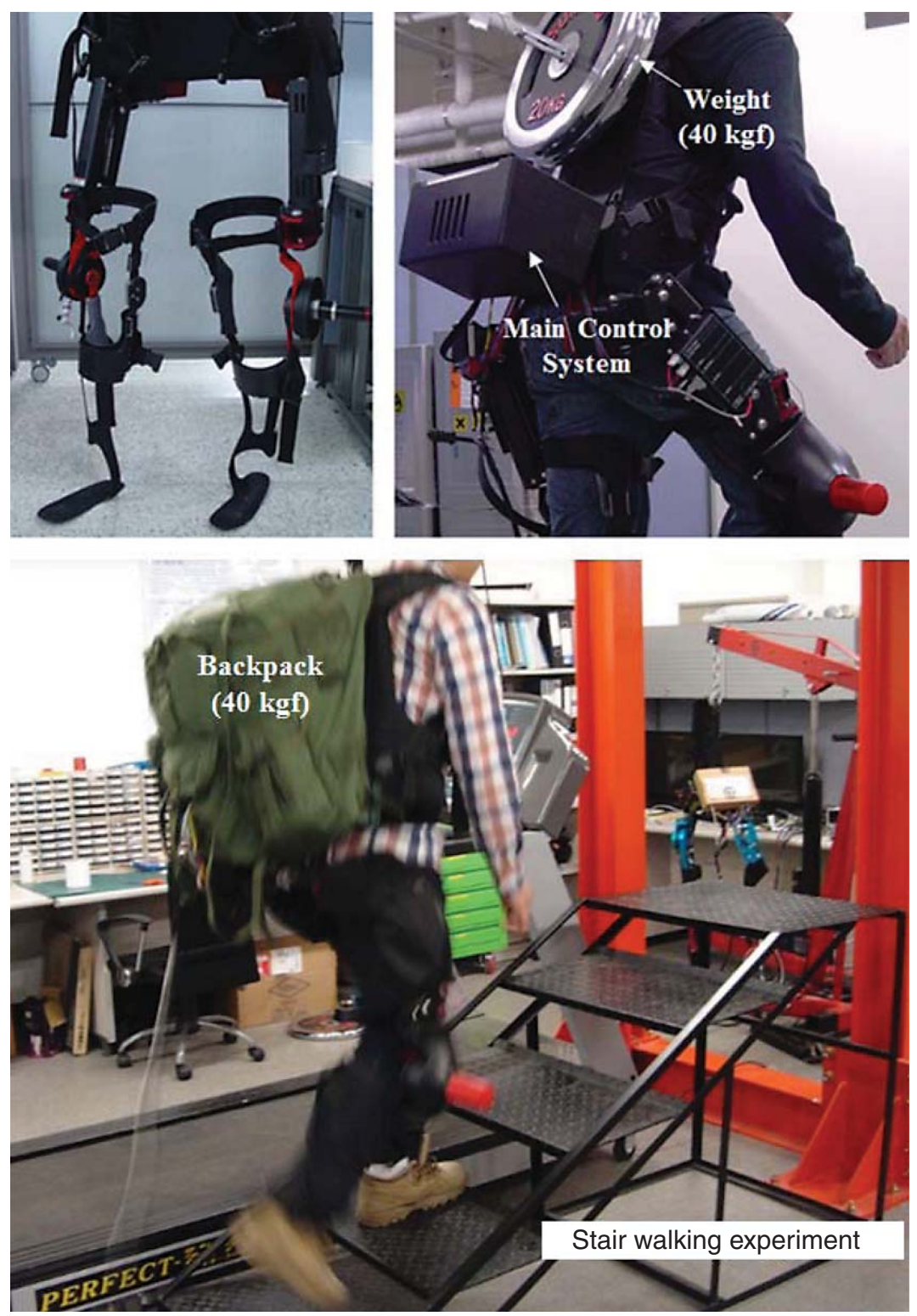

Fig. 11. Evaluation of the walking performance of the exoskeleton system ( $40 \mathrm{~kg} f$ of weight loaded).

\section{Conclusion and considerations}

This study describes considerations for developing the wearable lower extremity exoskeleton system for infantry soldiers, and based on existing R\&D cases, appropriate design criteria were proposed. They are summarized in the following.

- Basically, because infantry soldiers carry a variety of equipment, for an additionally worn exoskeleton system, the interference with existing equipment and additional burden on wearers should be minimized.

- For the TJMs of hip and ankle joints and multipledegree-of-freedom joints, the active joints of the exoskeleton are avoided, whereas the passive joints, which are focused on the transfer and distribution of the weight connected to the adjacent joints, are applied. 


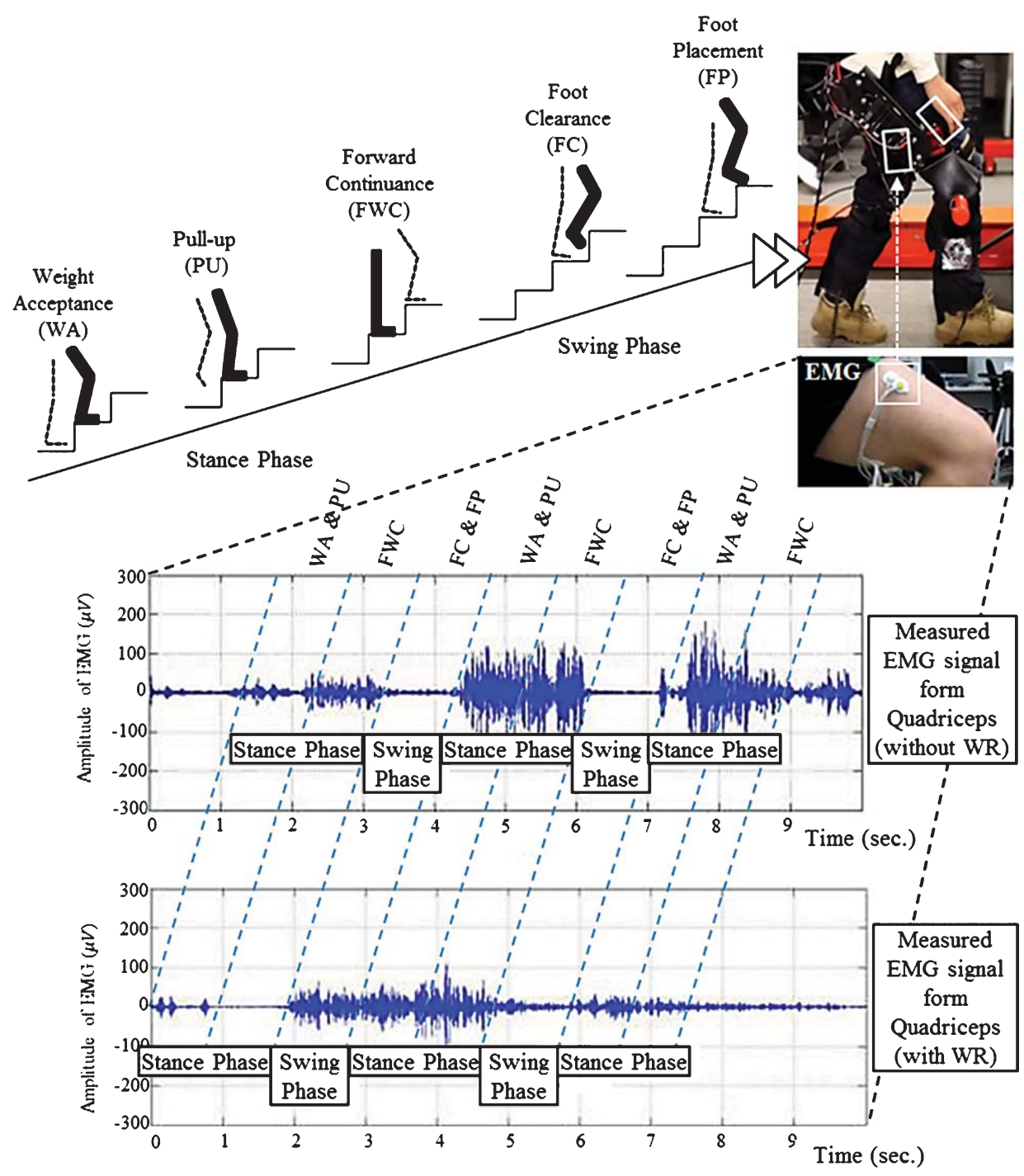

Fig. 12. Measurement results of the muscular activity of the wearer's quadriceps before/after wearing the exoskeleton system (Power assisting ratio $\cong 0.5$, Angular velocity of a knee joint $=30 \mathrm{rpm}$, WR: Wearable Robot).

- Walking on flat land, as shown in recent studies, can be performed by only supporting weight in the gravitational direction; however, in the case of mountainous terrain, active joints are necessarily required for walking over rough and sloped terrain. For this, it is thought that the underactuated exoskeleton system is appropriate: passive joints are applied to hip and ankle joints and active joints are applied to knee joints, which use the dynamic coupling effect of the link structure and muscular activation patterns while going up and down stairs.
- Although monoaxial active joints can be actuated by applying a force sensor or muscle volume sensor, which has recently been introduced, because infantry soldiers require various tactical motions, the responsiveness of the exoskeleton system to the various postures and motions of wearers should be improved by applying a foot sensor, clutch, and mechanism for adjusting the stiffness.

- Based on the above propositions, the prototype of the exoskeleton system was developed and presented, and its validity was verified by a simple 
stair walking experiment. The actuation performance and wearability in open fields will be verified by lightening the weight of the system and implementing an independent power system in further studies; the firearm rack system using the WSM proposed in this study will also be proposed.

\section{References}

[1] J. Knapik, E. Harman and K. Reynolds, Load carriage using packs: A review of physiological, biomechanical and medical aspects, Applied ergonomics 27 (1996), 207-216.

[2] J. Bachkosky, M. Andrews, R. Douglass, J. Feigley, L. Felton, F. Fernandez, P. Fratarangelo, A. Johnson-Winegar, R. Kohn, N. Polmar, R. Rumpf, J. Sommerer and W. Williamson Lightening the Load, Arlington, VA, Naval Research Advisory Committee 2007.

[3] S.A. Birrell, R.H. Hooper and R.A. Haslam, The effect of military load carriage on ground reaction forces, Gait \& Posture 26 (2007), 611-614.

[4] R. M. Orr, R. Pope, V. Johnston and J. Coyle, Load carriage: Minimizing soldier injuries through physical conditioning-A narrative review, Military and Veterans' Health 18 (2010), 31-38.

[5] B.H. Jones, B.C. Hansen, K.R. Kaufman and S. Brodine, Injuries in the military: A hidden epidemic, Falls Church, VA, Armed Forces Epidemiological Board, 1996.

[6] D.N. Cowan, B.H. Jones and R.A. Shaffer, Musculoskeletal injuries in the military training environment, Textbooks of Military Medicine (2003), 195-210.

[7] http://www.lockheedmartin.com/us/products/hulc.html (Last access: August 9th, 2014).

[8] http://raytheon.mediaroom.com (Last access: August 9th, 2014)

[9] http://www.cyberdyne.jp (Last access: August 9th, 2014).

[10] H.S. Moon, Present and Future of Wearable Robots, KISTI report, 2012

[11] C.J. Walsh, Biomimetic Design of an Under-Actuated Leg Exoskeleton for Load-Carrying Augmentation, PhD Thesis, Dept. of Mech. Eng, MIT, 2006.

[12] K.N. Gregorczyk, L. Hasselquist, J.M. Schiffman, C.K. Bensel, J.P. Obusek and D.J. Gutekunst, Effects of a lower-body exoskeleton device on metabolic cost and gait biomechanics during load carriage, Ergonomics 53 (2010), 1263-1275.

[13] W.S. Kim, S.H. Lee, M.S. Kang, J.S. Han and C.S. Han, Energy-efficient gait pattern generation of the powered robotic exoskeleton using DME, IEEE/RSJ International Conference on Intelligent Robots and Systems (2010), 2475-2480.

[14] J.M. Donelan, R. Kram and A.D. Kuo, Mechanical work for step-to-step transitions is a major determinant of the metabolic cost of human walking, Experimental Biology 205 (2002), 3717-3727.

[15] http://www.peterpaulmuller.com/thesis/ (Last access: August 9th, 2014)

[16] http://www.combatreform.org/combatlight.htm (Last access: August 9th, 2014).
[17] http://peosoldier.armylive.dodlive.mil/2010/06/28/equipmentpiece-of-the-week-3/ (Last access: August 9th, 2014).

[18] http://www.army.gov.au/Our-work/Equipment-and-clothing/ Soldier-Combat-Ensemble/Large-Field-Pack (Last access: August 9th, 2014)

[19] M.Q. Feng, Sensor Suits for Human Motion Detection, Report No: UCI-CEE-03-F03, California Univ. Irvine, 2006.

[20] S. Moromugi, Y. Koujina, S. Ariki, A. Okamoto, T. Tanaka M.Q. Feng and T. Ishimatsu, Muscle stiffness sensor to control an assistance device for the disabled, Artificial Life and Robotics 8 (2004), 42-45.

[21] S.N. Yu, H.D. Lee, S.H. Lee, W.S. Kim, J.S. Han and C.S. Han, Design of an under-actuated exoskeleton system for walking assist while load carrying, Advanced Robotics 26 (2012), 561-580.

[22] K.W. Hollander, P. Clouse, N. Cahill, A. Boehler, T.G. Sugar and A. Ayyar, Design of the orthotic load assistance device (OLAD), 7th Annual Dynamic Walking Conference (2012), 172-173.

[23] A.E. Patla and S.D. Prentice, The role of active force and intersegmental dynamics in the control of limb trajectory over obstacles during locomotion in humans, Exp Brain Res 106 (1995), 499-504.

[24] M. Bergerman, C. Lee and Y. Xu, Dynamic coupling of underactuated manipulators, Proceedings of the 4th IEEE Conference on Control Applications, Albany, USA, (1995), 500-505.

[25] Y.L. Gu and Y. Xu, Under-actuated robot systems: Dynamic interaction and adaptive control, Systems, Man, and Cybernetics, 1994. Humans, Information and Technology, IEEE International Conference on, (1994), 958-963.

[26] S.N. Yu, S.Y. Lee and C.S. Han, Methodology for the kinematical selection of a manipulator for a specified task, Autonomous Robots 23 (2007), 243-253.

[27] M.S. Cherry, Design and Evaluation of Elastic Exoskeletons for Human Running, PhD. Dissertation, University of Michigan 2010.

[28] R. Kram, Load Lugging Locomotion: Lessons from Indigenous People, Rhino Beetles, and Wallabies, Soldier Mobility: Innovations in Load Carriage System Design and Evaluation (2001)

[29] R.C. Juvinall and K.M. Marshek, Fundamentals of machine component design, Hoboken, NJ, John Wiley \& Sons, 2012, Chap.12.

[30] M.H. Lee, Development of walking assistance system with adjustive leg stiffness, MS Thesis, KAIST 2011.

[31] G.W. Brown, Equipoising support apparatus, US Patent No: 07618016, Issue date: Nov 17, 2009.

[32] J.P. Obusek, E.A. Harman, P.N. Frykman, C.J. Palmer and R.K. Bills, The relationship of backpack center of mass to the metabolic cost of load carriage, Med Sci Sports Exerc 29 (1997), 205.

[33] J. Knapik, Physiological, Biomechanical and Medical Aspects of Soldier Load Carriage, RTO Human Factors and Medicine Panel (HFM) Specialists' Meeting, Kingston, Canada, (2000), KN1-1 to KN1-14.

[34] A. Zoss and H. Kazerooni, Design of an electrically actuated lower extremity exoskeleton, Advanced Robotics 20 (2006), 967-988.

[35] J.A. Duncan, D.L. Kowalk and C.L. Vaughan, Six degree of freedom joint power in stair climbing, Gait and Posture 5 (1997), 204-210. 
[36] V.H. Frankel and A.H. Burstein, Orthopaedic biomechanics, Lea \& Febiger, 1970

[37] R. Drillis, R. Contini and M. Bluestein, Body segment parameters, Artificial Limbs 8 (1964), 44-66.
[38] A.R. Seo, H.Y. Jang, W.S. Kim, C.S. Han and J.S. Han, Development and verification of a volume sensor for measuring human behavior, Precision Engineering and manufacturing 13 (2012), 899-904. 

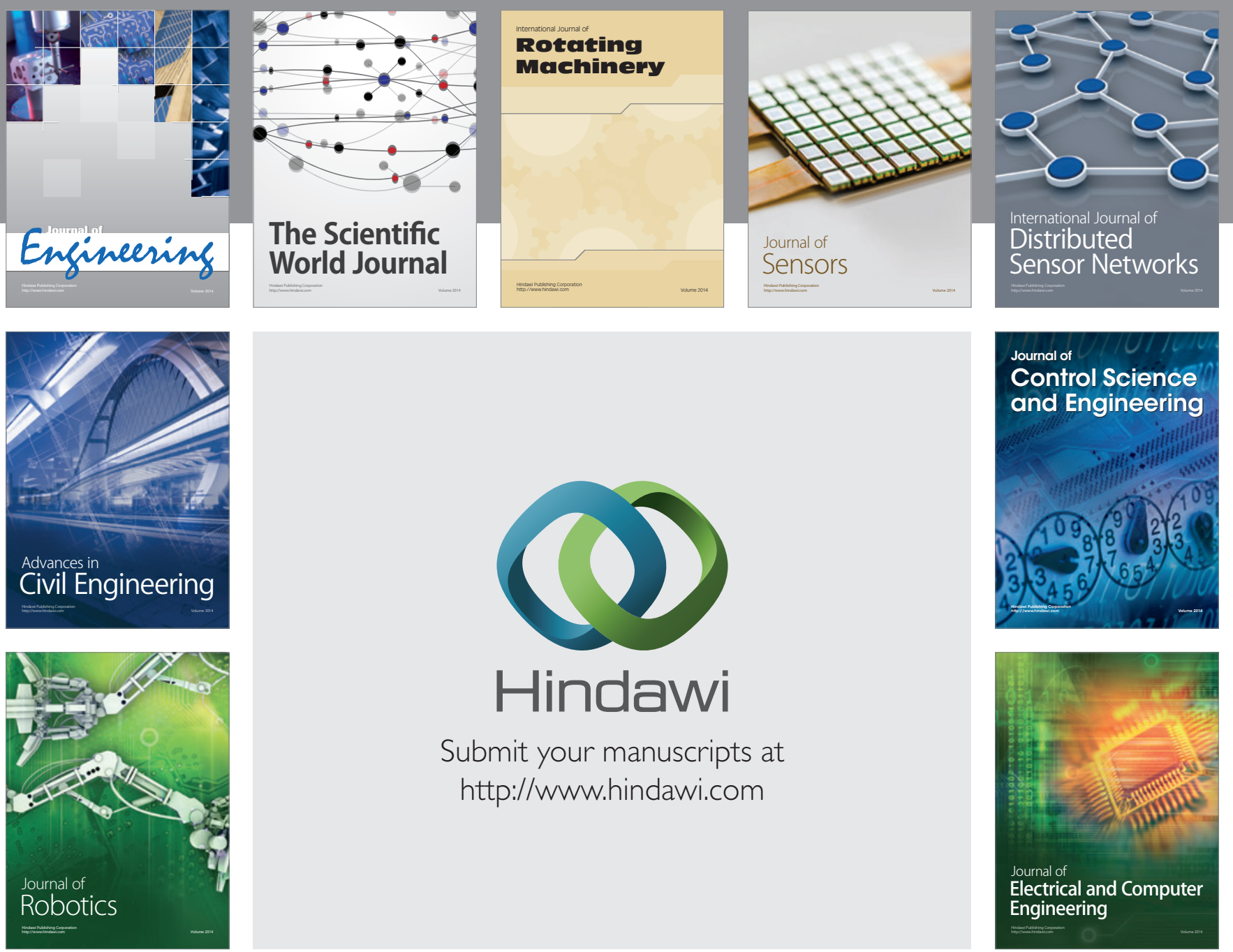

Submit your manuscripts at

http://www.hindawi.com
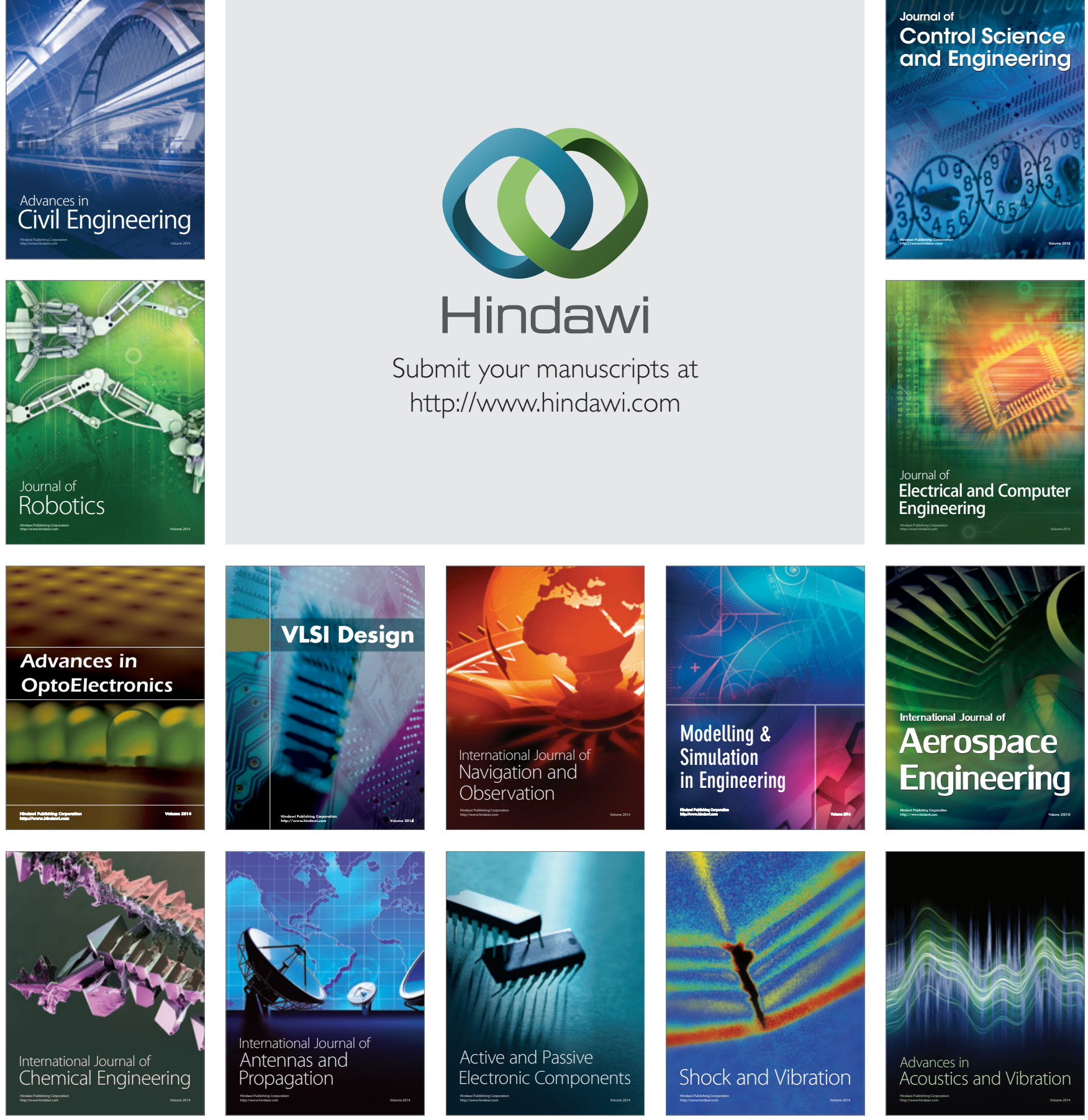